\title{
Dynamics of optical forces and torques in plasmonic systems: a surface integral equation
}

Kiselev, Andrei, Achouri, Karim, Martin, Olivier J.

Andrei Kiselev, Karim Achouri, Olivier J. F. Martin, "Dynamics of optical forces and torques in plasmonic systems: a surface integral equation," Proc. SPIE 11463, Optical Trapping and Optical Micromanipulation XVII, 1146313 (20 August 2020); doi: 10.1117/12.2567651

SPIE. Event: SPIE Nanoscience + Engineering, 2020, Online Only 


\title{
Dynamics of optical forces and torques in plasmonic systems: a surface integral equation
}

\author{
Andrei Kiselev*, Karim Achouri and Olivier J. F. Martin† \\ Nanophotonics and Metrology Laboratory, Swiss Federal Institute of Technology Lausanne (EPFL), \\ 1015 Lausanne, Switzerland
}

\begin{abstract}
A novel approach is introduced to determine the time evolution of optical forces and torques on arbitrary shape nanostructures by combining Maxwell's stress tensor with the surface integral equation method (SIE). Conventional time averaging of Maxwell's stress tensor allows obtaining an elegant form in terms of surface currents for the force exerted on nanostructures. Unfortunately, the information about the time dependence of the force - which can be very important in ultrafast photonics experiments and in nano-manipulation applications - is lost in such an approach. To overcome this, we have developed a time-domain method based on the inverse Fourier transform of the frequency-domain SIE. The calculations in the frequency domain allow accurately taking into account the dispersion of the permittivity function of the system and the use of surface currents enables the rigorous treatment of intricate geometries for the scatterer. Furthermore, the integration of Maxwell's stress tensor directly on the scatterer's boundary significantly reduces the required computation time and increases the accuracy of the method.

We show quite unusual sum frequency-like terms in the dynamics of the force appearing in Maxwell's stress tensor, which normally vanish for the time-averaged force. To illustrate this effect, we study how the pulse duration influences the dynamics of optical force in the case of a rectangular shape and Gaussian pulses illuminating thin film at normal incidence. In the framework of the developed numerical method, we study the influence of the sum-frequency-like terms on the dynamics of optical forces in the case of a spherical scatterer.
\end{abstract}

Keywords: Optical forces, surface integral approach, optical force dynamics, wave interference

\section{INTRODUCTION}

After the discovery of the optical trapping by Ashkin, ${ }^{1,2}$ a series of works have been dedicated to the various possibilities of manipulating materials, biological specimens and atoms with light. ${ }^{3-9}$ Continuous-wave (CW) illumination has become instrumental in developing these experiments. Indeed, knowing the resonances of the objects and being able to tune the excitation wavelength accordingly, one can achieve very high optical forces, especially close to the resonances of the structure. ${ }^{10-13}$ The majority of these experiments rely on employing time-averaged optical forces appearing at selected frequency.

Interestingly, the effects resulting from pulsed illumination are quite intricate and can substantially change the optical force. ${ }^{14-22}$ Despite the fact that all these works employ a very short laser pulse, fundamental physics that explains the values of the optical force in each particular pulsed-illumination experiment is surprisingly different. For example, pulsed illumination can be used to achieve very high peak power intensities, thus requiring less optical power than CW excitation to detach particles from a surface. ${ }^{18,19,22}$ In pulsed regime, a nonlinear Kerr effect can change the stiffness of the potential well in optical trapping experiments by utilizing the nonlinearity of the nanoparticle ${ }^{23-26}$ or the surrounding. ${ }^{27}$ Heating effects can also change the dielectric permittivity of the particle, thus enabling significantly larger optical forces. ${ }^{28}$ Femtosecond optical tweezers may be used to significantly enhance two-photon fluorescence compared to CW illumination. ${ }^{15,19,29}$

Complex physics appears during the interaction of an optical pulse with an object. Despite the fact that it was shown that the average force is independent on pulse duration, ${ }^{30}$ the time-domain dynamics of the force within the pulse is quite intricate and strongly depends on the pulse duration. ${ }^{14,30,31}$

*andrei.kiselev@epfl.ch, †olivier.martin@epfl.ch 
In this paper, we provide a pedagogical explanation of the emergence of the peak force in pulsed experiments. In Secs. 2.1-2.3, we present theoretical models that explain the enhancement of the peak force for rectangular or Gaussian illumination pulses acting on a thin film and show how the peak force depends on the pulse duration. In Sec. 2.4, we consider two specific frequencies from the pulse and show that concurrent illumination with two planewaves at different frequencies leads to the peak optical force enhancement. In Sec. 2.5, we propose a numerical method based on the surface integral equation to numerically calculate the force in the case of a two-wave illumination. In Sec. 2.6, we apply this method to calculate the dynamics of the optical force acting on a $150 \mathrm{~nm}$ silver sphere.

\section{RESULTS}

\subsection{Scaling peak optical force with the laser bandwidth}

In this section, we discuss the effect appearing due to the interaction between different frequencies of the illumination spectrum and characterize the unusual enhanced peak force, which appears for broader laser spectra.

Let us consider the optical force acting on a metallic thin film placed in vacuum and illuminated by a plane wave pulse with electric and magnetic fields $\mathbf{E}_{0}(t)$ and $\mathbf{H}_{0}(t)$ in the geometry illustrated in Figure 1. The dynamics of the optical force due to the applied electromagnetic field can be found using Maxwell's stress tensor, which is defined as ${ }^{32}$

$$
\mathbf{F}(t)=\int_{S} \overline{\mathbf{T}}(t) \mathbf{n} d S-\varepsilon_{0} \mu_{0} \frac{d}{d t} \int_{V} \varepsilon_{V} \mu_{V} \mathbf{S}(t) d V
$$

where $\overline{\mathbf{T}}(t) \mathbf{n}$ is the time-varying projection of Maxwell's stress tensor on the outward normal $\mathbf{n}$ to the closed surface $S$

$$
\overline{\mathbf{T}}(t) \mathbf{n}=\varepsilon_{0} \varepsilon_{s} \mathbf{E}(t)(\mathbf{E}(t) \cdot \mathbf{n})+\mu_{0} \mu_{s} \mathbf{H}(t)(\mathbf{H}(t) \cdot \mathbf{n})-\frac{1}{2}\left(\varepsilon_{0} \varepsilon_{s}(\mathbf{E}(t) \cdot \mathbf{E}(t))+\mu_{0} \mu_{s}(\mathbf{H}(t) \cdot \mathbf{H}(t))\right) \mathbf{n},
$$

and $\varepsilon_{V}, \mu_{V}\left(\varepsilon_{s}, \mu_{s}\right)$ are permittivity and permeability of the object (surrounding medium); while the Poynting vector $\mathbf{S}(t)=\mathbf{E}(t) \times \mathbf{H}(t)$ is integrated inside the volume $V$ of the object. Henceforth, we will assume that the surrounding medium is vacuum with $\varepsilon_{s}=1$ and $\mu_{s}=1$.

In the configuration presented in Figure 1, we assume a negligibly small thickness of the plate. Thus, the integration over the entire surface $S$ in Eq. (1) can be substituted by the integration over the upper and lower sides of the plate. In addition, the volumetric integration of the Poynting vector in Eq. (1) vanishes due to the negligibly small volume of the film. In the case of normal incidence, the electric and magnetic components of the field are always parallel to the long side of the plates, thus, $\mathbf{E} \cdot \mathbf{n}=\mathbf{H} \cdot \mathbf{n}=0$. These assumptions significantly simplify Eqs. (1) and (2) leading to

$$
\mathbf{F}(t)=-\int_{S}^{1} \frac{1}{2}\left(\varepsilon_{0}(\mathbf{E}(t) \cdot \mathbf{E}(t))+\mu_{0}(\mathbf{H}(t) \cdot \mathbf{H}(t))\right) \mathbf{n} d S .
$$

The incident field is defined by the electric and magnetic components $E_{0}(t)$ and $H_{0}(t)$, as shown in the Figure 1 , while the fields of the reflected and transmitted waves are defined from the incident ones using the coefficients $r$ and $t$, respectively. For simplicity, in the following we assume no frequency dependence of $r$ and $t$. 


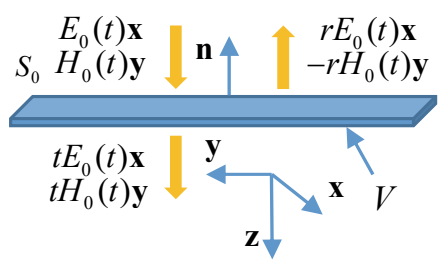

Figure 1. Sketch of a thin film illuminated by a pulse propagating along the $\mathbf{z}$-direction. The dynamics of the electric and magnetic fields for the incident pulse are defined by $E_{0}(t)$ and $H_{0}(t)$. Transmission and reflection coefficients $t$ and $r$ complete the definition of the field above and below the film.

The total electric field above the surface $\mathbf{E}_{\text {above }}(t)$ of the film has the form

$$
\mathbf{E}_{\text {above }}(t)=\mathbf{E}_{0}(t)(1+r)
$$

Recalling that the electric and magnetic fields of the planewave are related through the wavevector $\mathbf{k}$ as

$$
\mathbf{k} \times \mathbf{E}_{0} \sim \mathbf{H}_{0}
$$

the magnetic component of the reflected wave should be taken into account with the minus sign in the equation for the total magnetic field $\mathbf{H}_{\text {above }}(t)$ above the surface,

$$
\mathbf{H}_{\text {above }}(t)=\mathbf{H}_{0}(t)(1-r) \text {. }
$$

The electric and magnetic fields below the surface $\mathbf{E}_{\text {below }}(t)$ and $\mathbf{H}_{\text {below }}(t)$ are defined by

$$
\begin{aligned}
& \mathbf{E}_{\text {below }}(t)=\mathbf{E}_{0}(t) t, \\
& \mathbf{H}_{\text {below }}(t)=\mathbf{H}_{0}(t) t .
\end{aligned}
$$

The projection of the optical force along the z-axis in Eq. (3) then takes the form

$$
\mathbf{F}_{z}(t)=-\frac{1}{2}\left(\begin{array}{c}
\varepsilon_{0}\left(\mathbf{E}_{\text {below }}(t) \cdot \mathbf{E}_{\text {below }}(t)-\mathbf{E}_{\text {above }}(t) \cdot \mathbf{E}_{\text {above }}(t)\right)+ \\
\mu_{0}\left(\mathbf{H}_{\text {below }}(t) \cdot \mathbf{H}_{\text {below }}(t)-\mathbf{H}_{\text {above }}(t) \cdot \mathbf{H}_{\text {above }}(t)\right)
\end{array}\right) \mathbf{z} S_{0},
$$

where $S_{0}$ is the surface of the long side (up or down) of the film. Using Eqs. (4) - (8), the equation for the force (8) reduces to

$$
\mathbf{F}_{z}(t)=\frac{1}{2}\left(\begin{array}{c}
\varepsilon_{0} \mathbf{E}_{0}^{2}(t)\left((1+r)^{2}-t^{2}\right)+ \\
\mu_{0} \mathbf{H}_{0}^{2}(t)\left((1-r)^{2}-t^{2}\right)
\end{array}\right) \mathbf{z} \cdot S
$$

In the case of planewave propagation, the electric and magnetic components are related through the impedance of vacuum, ${ }^{33}$

$$
Z_{0}=\sqrt{\frac{\mu_{0}}{\varepsilon_{0}}}=\frac{|E|}{|H|} .
$$

Expressing the magnetic field in Eq. (10) using (10) leads to

$$
\mathbf{F}_{z}(t)=\varepsilon_{0} \mathbf{E}_{0}^{2}(t)\left(1+r^{2}-t^{2}\right) \mathbf{z} \cdot S
$$

This formula suggests that in the case of thin metal film, the force directly depends on the square of the instantaneous value of electric field on the surface of the film. In the following, we explain how different temporal spectra $\mathbf{E}_{0}(t)$ influence the peak force. 


\subsection{Peak optical force in the case of a rectangular shape pulse}

Let us assume that the illumination spectrum has a rectangular shape

With the inverse Fourier transform defined as

$$
S(\omega)=\left\{\begin{array}{l}
0, \text { if }|\omega|>\Delta \omega / 2 \\
E_{0} / 2 \sqrt{\Delta \omega}, \text { if }|\omega|=\Delta \omega / 2 . \\
E_{0} / \sqrt{\Delta \omega}, \text { if }|\omega|<\Delta \omega / 2
\end{array}\right.
$$

$$
E(t)=\frac{1}{\sqrt{2 \pi}} \int_{-\infty}^{+\infty} S(\omega) e^{-i \omega t} d t,
$$

the time-domain signal corresponding to Eq. (12) is

$$
E(t)=E_{0} \frac{\sqrt{\Delta \omega}}{\sqrt{2 \pi}} \frac{\sin (\Delta \omega t / 2)}{\Delta \omega t / 2} .
$$

The rectangular shape pulse spectrum as well as its time-domain counterpart are presented in Figure 2.
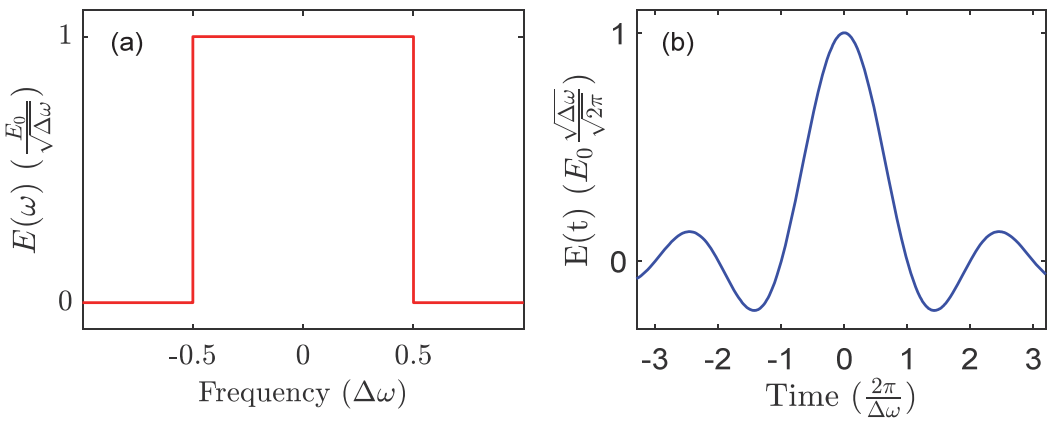

Figure 2. (a) Rectangular spectrum. (b) Corresponding sinc-shape time-domain response.

Note that the field in Eq. (14) is normalized so that the total energy of the pulse, given by

$$
P=\int_{-\infty}^{+\infty} E^{2}(t) d t=E_{0}^{2},
$$

does not depend on filter band pass $\Delta \omega$. The equation for the force dynamics is now obtained by substituting Eq. (12) into (14) as

$$
\mathbf{F}_{z}(t)=\varepsilon_{0}\left(E_{0} \frac{\sqrt{\Delta \omega}}{\sqrt{2 \pi}} \frac{\sin (\Delta \omega t / 2)}{\Delta \omega t / 2}\right)^{2}\left(1+r^{2}-t^{2}\right) \mathbf{z} \cdot S .
$$

This force takes its maximum value when $\mathrm{t}=0$, therefore, considering that

$$
\lim _{\Delta \omega t \rightarrow 0} \frac{\sin (\Delta \omega t / 2)}{\Delta \omega t / 2}=1,
$$

the maximum of the optical force dynamics can be simplified to

$$
\mathbf{F}_{z}^{\text {peak }}=\mathbf{F}_{z}(0)=\varepsilon_{0}\left(E_{0} \frac{\sqrt{\Delta \omega}}{\sqrt{2 \pi}}\right)^{2}\left(1+r^{2}-t^{2}\right) \mathbf{z} \cdot S \sim \Delta \omega .
$$

Interestingly, the peak force depends on the spectral width of the excitation. The total momentum transferred to the film, defined as 


$$
S(t)=\int_{-\infty}^{t} \mathbf{F}_{z}(t) d t
$$

can be applied to calculate the total momentum transferred during one pulse,

$$
S(+\infty)=\varepsilon_{0} E_{0}^{2}\left(1+r^{2}-t^{2}\right) \mathbf{z} \cdot S .
$$

As can be seen, the total transferred momentum does not depend on $\Delta \omega$, which means that the pulse duration cannot affect the average dynamics of the pulse, as was pointed out previously. ${ }^{30}$

\subsection{Peak optical force in the case of a Gaussian pulse}

In order to show how the pulse width affects the peak force for different kinds of pulses, we study the peak force resulting from the Gaussian pulse illumination defined by

$$
E(\omega)=\frac{E_{0} \sqrt{T}}{\sqrt{2 \sqrt{\pi}\left(1+\exp \left(-\omega_{0}^{2} T^{2}\right)\right)}}\left[\exp \left(-\left(\omega-\omega_{0}\right)^{2} \cdot T^{2} / 2\right)+\exp \left(-\left(\omega+\omega_{0}\right)^{2} \cdot T^{2} / 2\right)\right]
$$

where $\mathrm{T}$ defines the time width of the pulse. Note that the normalization in Eq. (22) is chosen such that the total energy of the pulse is the same as that in Eq. (16). Using the inverse Fourier transform defined by Eq. (14), the time-domain expression of the electric field (21) is given by

$$
E(t)=\frac{E_{0} \cos \left(\omega_{0} t\right) \exp \left(-t^{2} /\left(2 T^{2}\right)\right)}{\sqrt{T \sqrt{\pi}\left(1+\exp \left(-\omega_{0}^{2} T^{2}\right)\right) / 2}} .
$$

The Gaussian shape pulse spectrum as well as its time-domain counterpart are presented in Figure 3 for $\omega_{0} T=8 / \sqrt{2}$.
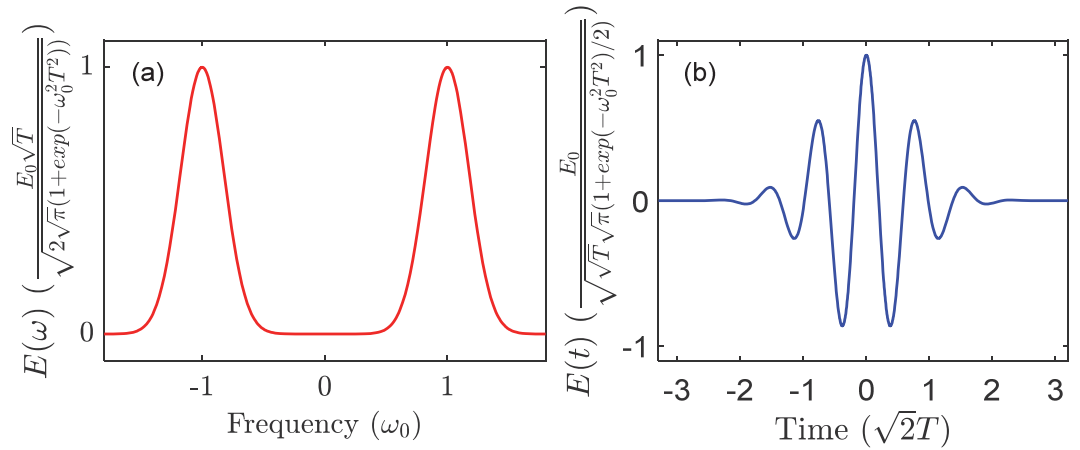

Figure 3. (a) Gaussian pulse spectrum. (b) Gaussian pulse electric field dynamics in time-domain.

The total momentum transferred to the film,

$$
S(+\infty)=\varepsilon_{0} E_{0}^{2}\left(1+r^{2}-t^{2}\right) \mathbf{z} \cdot S,
$$

also does not depend on the pulse width, as before. The peak force acting on a plate at $\mathrm{t}=0$, as in Eq. (19), is given by

$$
\begin{gathered}
\mathbf{F}_{z}^{\text {peak }}=\mathbf{F}_{z}(0)=\varepsilon_{0}\left(\frac{E_{0}}{\sqrt{T \sqrt{\pi}\left(1+\exp \left(-\omega_{0}^{2} T^{2}\right)\right) / 2}}\right)^{2}\left(1+r^{2}-t^{2}\right) \mathbf{z} \cdot S, \\
\mathbf{F}_{z}^{\text {peak }} \sim \frac{1}{T\left(1+\exp \left(-\omega_{0}^{2} T^{2}\right)\right)},
\end{gathered}
$$

Recalling that $1 / T \sim \Delta \omega$, the peak force scales again as 


$$
\mathbf{F}_{z}^{\text {peak }} \sim \Delta \omega .
$$

Overall, these results suggest that by increasing the bandwidth of the pulse, the peak optical force also increases. In the following section, we give a simple physical explanation for this phenomenon.

\subsection{Frequency interaction changes the peak force}

In order to get insights into the origin of peak force enhancement, let us first consider the interaction of two planewaves with frequencies $\omega_{1}$ and $\omega_{2}$. The optical force due to the two-wave interaction can be found from Eq. (21) and reads

$$
\mathbf{F}_{z}(t)=\varepsilon_{0}\left(E_{1} \sin \left(\omega_{1} t\right)+E_{2} \sin \left(\omega_{2} t\right)\right)^{2}\left(1+r^{2}-t^{2}\right) \mathbf{z} \cdot S,
$$

which can be further transformed into

$$
\mathbf{F}_{z}(t)=\varepsilon_{0}\left(\begin{array}{l}
\frac{E_{1}^{2}+E_{2}^{2}}{2}-\frac{E_{1}^{2}}{2} \cos \left(2 \omega_{1} t\right)-\frac{E_{2}^{2}}{2} \cos \left(2 \omega_{2} t\right)+ \\
E_{1} E_{2} \cos \left(\left[\omega_{1}-\omega_{2}\right] t\right)-E_{1} E_{2} \cos \left(\left[\omega_{1}+\omega_{2}\right] t\right)
\end{array}\right)\left(1+r^{2}-t^{2}\right) \mathbf{z} \cdot S
$$

As can be seen from Eq. (29), in addition to the oscillations at $\omega_{1}$ and $\omega_{2}$, we also have now oscillations at the sum frequency and the difference frequency. These terms, with amplitudes $E_{1} E_{2}$ modulate the instantaneous force. When we extend this to a pulse, built from many different frequencies, all the frequency components interfere between each other thus contributing to this beating effect and therefore significantly changing the force in the time-domain. Let us note that this phenomenon is akin to the generation of second harmonic under pulsed illumination. ${ }^{34-39}$

\subsection{Surface integral equation for the accurate calculation of the dynamics of the optical forces}

In this section, we discuss a numerical approach to find the dynamics of the optical force acting on arbitrary shape particle. Generally, numerical integration of Maxwell's stress tensor on the sphere enclosing the scatterer is required to find the optical force. In addition, the integration of the Poynting vector inside the volume of the particle is required to consider the variation of the momentum of the electromagnetic field. ${ }^{32}$ In order to find the fields, one needs to use numerical techniques based on Maxwell's equations. ${ }^{40,41}$ In this paper, we use the surface integral equation (SIE), which has been proven as a very good method for the solution of scattering problems thanks to its accuracy and low computation requirements. ${ }^{41,42}$ Also, being a frequency-domain method, this approach allows taking into account the dispersion of the dielectric function, which is more difficult for time-domain methods. ${ }^{41,42}$

Due to the freedom in choosing the integration surface in Eq. (1), we set $S$ to be the surface of the scatterer. In this case, the volumetric integration of the Poynting vector $\mathbf{S}(t)$ is performed within the volume of the structure. In the framework of the surface integral equation formulation, the fields at the surface of the object are represented in terms of so-called surface currents, which in harmonic representation $e^{-i \omega t}$ are related to the complex amplitudes of the electric $\mathbf{E}(\mathbf{r})$ and magnetic $\mathbf{H}(\mathbf{r})$ fields on the surface of the scatterer: ${ }^{42}$

$$
\begin{gathered}
\mathbf{J}(\mathbf{r})=\mathbf{n} \times \mathbf{H}(\mathbf{r}), \mathbf{r} \in S \\
\mathbf{M}(\mathbf{r})=-\mathbf{n} \times \mathbf{E}(\mathbf{r}) .
\end{gathered}
$$

Recalling the main results from Ji et al ${ }^{43}$ and Kern et al., ${ }^{42}$ the dynamics of the electric and magnetic fields on the scatterer's surface at frequency $\omega$ can be found through a separation into normal and perpendicular components as

$$
\begin{gathered}
\mathbf{E}_{\perp}(\mathbf{r}, t)=\operatorname{Re}\left[-\frac{i}{\omega \varepsilon_{V}(\omega) \varepsilon_{0}}(\nabla \cdot \mathbf{J}(\mathbf{r})) e^{-i \omega t}\right] \mathbf{n}, \\
\mathbf{E}_{\|}(\mathbf{r}, t)=\operatorname{Re}\left[[\mathbf{n} \times \mathbf{M}(\mathbf{r})] e^{-i \omega t}\right] . \\
\mathbf{H}_{\perp}(\mathbf{r}, t)=\operatorname{Re}\left[-\frac{i}{\omega \mu_{0} \mu_{V}(\omega)}(\nabla \cdot \mathbf{M}(\mathbf{r})) e^{-i \omega t}\right] \mathbf{n},
\end{gathered}
$$




$$
\mathbf{H}_{\|}(\mathbf{r}, t)=\operatorname{Re}\left[[\mathbf{J}(\mathbf{r}) \times \mathbf{n}] e^{-i \omega t}\right] .
$$

Here $\varepsilon_{V}(\omega)$ and $\mu_{V}(\omega)$ are dielectric permittivity and magnetic permeability of the scatterer at frequency $\omega$. Assuming that the scatterer is a homogeneous body with a surface $S$, the electric and magnetic fields inside the volume of the scatterer read

$$
\begin{aligned}
& \mathbf{E}(\mathbf{r}, t)=-i \omega \mu_{V}(\omega) \int_{S} d S^{\prime} \overline{\mathbf{G}}\left(\mathbf{r}, \mathbf{r}^{\prime}\right) \cdot \mathbf{J}\left(\mathbf{r}^{\prime}\right) e^{-i \omega t}-\int_{S} d S^{\prime}\left[\nabla^{\prime} \times \overline{\mathbf{G}}\left(\mathbf{r}, \mathbf{r}^{\prime}\right)\right] \cdot \mathbf{M}\left(\mathbf{r}^{\prime}\right) e^{-i \omega t}, \mathbf{r} \in V, \\
& \mathbf{H}(\mathbf{r}, t)=-i \omega \varepsilon_{V}(\omega) \int_{S} d S^{\prime} \overline{\mathbf{G}}\left(\mathbf{r}, \mathbf{r}^{\prime}\right) \cdot \mathbf{M}\left(\mathbf{r}^{\prime}\right) e^{-i \omega t}+\int_{S} d S^{\prime}\left[\nabla^{\prime} \times \overline{\mathbf{G}}\left(\mathbf{r}, \mathbf{r}^{\prime}\right)\right] \cdot \mathbf{J}\left(\mathbf{r}^{\prime}\right) e^{-i \omega t} .
\end{aligned}
$$

With the dyadic Green's function defined as

$$
\overline{\mathbf{G}}_{i}\left(\mathbf{r}, \mathbf{r}^{\prime}\right)=\left(\overline{\mathbf{1}}+\frac{\nabla \nabla}{k^{2}}\right) \frac{\exp (i k R)}{4 \pi R},
$$

where $k=\left(\omega^{2} \varepsilon_{V}(\omega) \mu_{V}(\omega)\right)^{1 / 2}$ is the wavevector inside the scatterer and $R=\left|\mathbf{r}-\mathbf{r}^{\prime}\right|$. Let us introduce parallel and perpendicular components of electric and magnetic fields with respect to the local surface normal vector $\mathbf{n}$

$$
\begin{aligned}
\mathbf{E}(\mathbf{r}, t) & =\mathbf{E}_{\perp}(\mathbf{r}, t)+\mathbf{E}_{\|}(\mathbf{r}, t), \\
\mathbf{H}(\mathbf{r}, t) & =\mathbf{H}_{\perp}(\mathbf{r}, t)+\mathbf{H}_{\|}(\mathbf{r}, t) .
\end{aligned}
$$

Inserting Eqs. (39), (40) into Eq. (2) gives:

$$
\begin{aligned}
& \overline{\mathbf{T}}(t) \mathbf{n}=\varepsilon_{0} \varepsilon_{V}(\omega) \mathbf{E}(\mathbf{r}, t)\left(\mathbf{E}_{\perp}(\mathbf{r}, t) \cdot \mathbf{n}\right)+\mu_{0} \mu_{V}(\omega) \mathbf{H}(\mathbf{r}, t)\left(\mathbf{H}_{\perp}(\mathbf{r}, t) \cdot \mathbf{n}\right)+ \\
& -\frac{1}{2}\left(\varepsilon_{0} \varepsilon_{V}(\omega)\left(\mathbf{E}_{\perp}(\mathbf{r}, t) \cdot \mathbf{E}_{\perp}(\mathbf{r}, t)+\mathbf{E}_{\|}(\mathbf{r}, t) \cdot \mathbf{E}_{\|}(\mathbf{r}, t)\right)+\mu_{0} \mu_{V}(\omega)\left(\mathbf{H}_{\perp}(\mathbf{r}, t) \cdot \mathbf{H}_{\perp}(\mathbf{r}, t)+\mathbf{H}_{\|}(\mathbf{r}, t) \cdot \mathbf{H}_{\|}(\mathbf{r}, t)\right)\right) \mathbf{n} .
\end{aligned}
$$

In the case of a two-wave illumination at frequencies $\omega_{1}$ and $\omega_{2}$, the components of the electric field require slight modification as

$$
\begin{gathered}
\mathbf{E}_{\perp}(\mathbf{r}, t)=\operatorname{Re}\left[-\frac{i}{\omega_{1} \varepsilon_{V}\left(\omega_{1}\right) \varepsilon_{0}}\left(\nabla \cdot \mathbf{J}_{1}(\mathbf{r})\right) e^{-i \omega_{1} t}+-\frac{i}{\omega_{2} \varepsilon_{V}\left(\omega_{2}\right) \varepsilon_{0}}\left(\nabla \cdot \mathbf{J}_{2}(\mathbf{r})\right) e^{-i \omega_{2} t}\right] \hat{\mathbf{n}},(42) \\
\mathbf{E}_{\|}(\mathbf{r}, t)=\operatorname{Re}\left[\left[\mathbf{n} \times \mathbf{M}_{1}(\mathbf{r})\right] e^{-i \omega_{1} t}+\left[\mathbf{n} \times \mathbf{M}_{2}(\mathbf{r})\right] e^{-i \omega_{2} t}\right]
\end{gathered}
$$

And for the magnetic field

$$
\begin{gathered}
\mathbf{H}_{\perp}(\mathbf{r}, t)=\operatorname{Re}\left[-\frac{i}{\omega \mu_{0} \mu_{V}\left(\omega_{1}\right)}\left(\nabla \cdot \mathbf{M}_{1}(\mathbf{r})\right) e^{-i \omega_{1} t}-\frac{i}{\omega \mu_{0} \mu_{V}\left(\omega_{2}\right)}\left(\nabla \cdot \mathbf{M}_{2}(\mathbf{r})\right) e^{-i \omega_{2} t}\right] \hat{\mathbf{n}} \\
\mathbf{H}_{\|}(\mathbf{r}, t)=\operatorname{Re}\left[\left[\mathbf{J}_{1}(\mathbf{r}) \times \mathbf{n}\right] e^{-i \omega_{1} t}+\left[\mathbf{J}_{2}(\mathbf{r}) \times \mathbf{n}\right] e^{-i \omega_{2} t}\right]
\end{gathered}
$$

By inserting Eqs. (42)-(45) into Eq. (41), the dynamics of the force attributed to Maxwell's stress tensor can be obtained for the case of a two-wave illumination. The integration of the Poynting vector inside the volume of the object can also be simplified by writing the fields in frequency domain:

$$
\mathbf{E}(\mathbf{r}, t)=2 \operatorname{Re}\left[\mathbf{E}_{1}(\mathbf{r}) e^{-i \omega_{1} t}+\mathbf{E}_{2}(\mathbf{r}) e^{-i \omega_{2} t}\right], \mathbf{r} \in V
$$




$$
\frac{d \mathbf{E}(\mathbf{r}, t)}{d t}=2 \operatorname{Re}\left[-i \omega_{1} \mathbf{E}_{1}(\mathbf{r}) e^{-i \omega_{1} t}-i \omega_{2} \mathbf{E}_{2}(\mathbf{r}) e^{-i \omega_{2} t}\right] .
$$

By replacing $\mathbf{E}$ and $\mathbf{H}$ in Eqs. (46) and (47), the same expressions can be obtained for magnetic field thus enabling finding the derivative of the Poyting vector. Thus, using Eqs. (36), (37), (46) and (47) the dynamics of the force due to the exchange of electric field momentum can be obtained. Note that the errors in the volumetric calculation of the Poynting vector in Eqs. (36) and (37) due to the divergence of the Green's tensor in the vicinity of the scatterer's surface for $R=\left|\mathbf{r}-\mathbf{r}^{\prime}\right| \rightarrow 0$ can be avoided by using the singularity subtraction technique. ${ }^{44,45}$

\subsection{Dynamics of the optical forces acting on a sphere}

In this section, we apply the method developed in Sec. 2.5 to study the dynamics of optical forces acting on a $150 \mathrm{~nm}$ diameter silver sphere for an illumination with two planewaves at frequencies $\omega_{1}$ and $\omega_{2}$. Before discussing the dynamics caused by two-wave illumination, let us look at the force emerging due to single frequency illumination. In Figure 4(a), we present the time-average optical force at different frequencies. From Figure 4(a), we selected two frequencies at $\omega_{1}=2.67 \mathrm{eV}$ corresponding to the maximum of the average force and at $\omega_{2}=2.13 \mathrm{eV}$, slightly below, to obtain a beating effect. The dynamics of optical forces at frequencies $\omega_{1}$ and $\omega_{2}$ is represented in Figure 4(b) as $\mathrm{F}_{1}(t)$ and $\mathrm{F}_{2}(t)$. As can be seen, the peak optical force reaches the maximum values of $6.1 \cdot 10^{-22} \mathrm{~N} /\left(\mathrm{W} / \mathrm{m}^{2}\right)$ and $4.8 \cdot 10^{-22}$ $N /\left(\mathrm{W} / \mathrm{m}^{2}\right)$ for the frequencies $\omega_{1}$ and $\omega_{2}$, respectively. Quite interestingly, simultaneous illumination with the two waves at these frequencies leads to the emergence of the additional beating component of the optical force, as described in Sec. 2.8. The dynamics of this component oscillating at the beating frequencies $\omega_{1}-\omega_{2}$ and $\omega_{1}+\omega_{2}$ is presented in Figure 4(c). Note that the amplitude of the beating force oscillations is $11 \cdot 10^{-22} \mathrm{~N} /\left(\mathrm{W} / \mathrm{m}^{2}\right)$ in this case and even higher than the amplitudes of the oscillations of $\mathrm{F}_{1}(t)$ and $\mathrm{F}_{2}(t)$. Consequently, the dynamics of the optical force under two waves illumination $\mathrm{F}_{\text {sum }}(t)$ equals to $\mathrm{F}_{1}(t)+\mathrm{F}_{2}(t)+\mathrm{F}_{\text {beating }}(t)$ and is shown in Figure 4(c). 

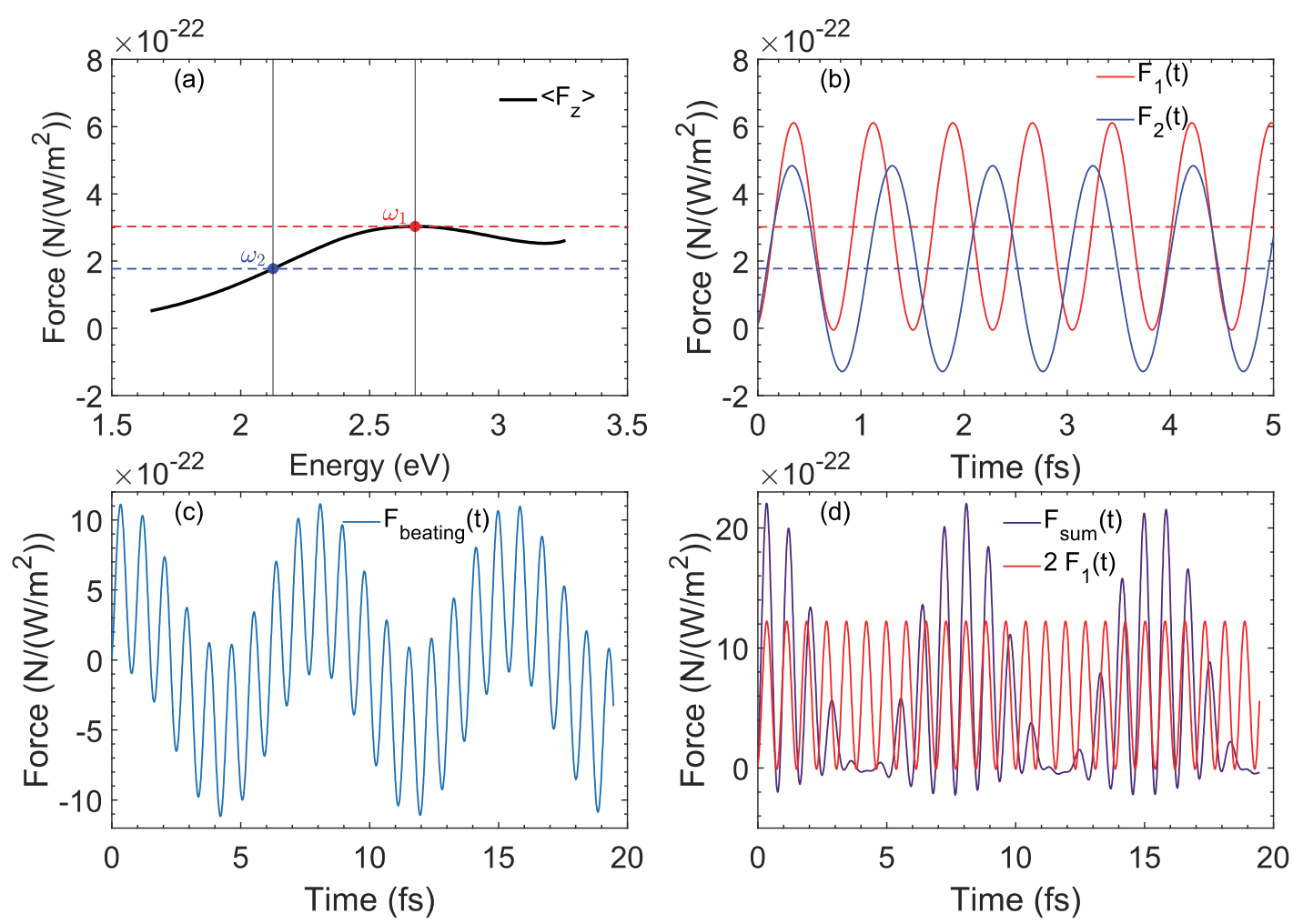

Figure 4. Time-domain analysis of the optical force acting on a $150 \mathrm{~nm}$ diameter silver sphere in vacuum under two-wave illumination. (a) Optical force under monochromatic illumination. (b) Dynamics of the optical force at frequencies $\omega_{1}=2.67 \mathrm{eV}$ and $\omega_{2}=2.13 \mathrm{eV}$. (c) Dynamics of the beating component in the case of simultaneous illumination with the two waves. (d) Dynamics of the optical force under two-wave illumination (purple) and dynamics of the optical force under single-wave illumination (red).

Comparison of the two-wave illumination force with the $\mathrm{F}_{1}(t)$ shows significant enhancement of the amplitude of the two-wave force. In Figure 4(c), we multiplied the force $\mathrm{F}_{1}(t)$ by the factor of two to insure equal power of incident illumination of the single wave and two wave illuminations.

\section{CONCLUSION}

We have discussed the instantaneous optical force acting on a thin film illuminated by rectangular or Gaussian pulses at normal incidence. Keeping the average power of the pulse constant for varying spectral widths, we have observed the enhancement of the peak force for pulses with largest bandwidth. We have further shown that the appearance of a beating effect, associated with the different spectral components of the illumination pulse, explains this enhancement of the peak force. Finally, we have proposed an accurate numerical method to compute the variations of the optical force in the time domain, based on a frequency-domain surface integral equation method. This method was applied to calculate the peak force enhancement for a $150 \mathrm{~nm}$ sphere illuminated by two waves with different frequencies.

\section{ACKNOWLEDGMENTS}

Funding from the European Research Council (ERC-2015-AdG-695206) is gratefully acknowledged. 


\section{REFERENCES}

1. A. Ashkin, J. M. Dziedzic, J. E. Bjorkholm, and S. Chu, "Observation of a single-beam gradient force optical trap for dielectric particles," Opt. Lett. 11(5), 288-290 (1986).

2. A. Ashkin, "Acceleration and Trapping of Particles by Radiation Pressure," Phys. Rev. Lett. 24(4), 156-159 (1970).

3. C. Bradac, "Nanoscale Optical Trapping: A Review," Adv. Opt. Mater. 6(12), 1800005 (2018).

4. D. Gao, W. Ding, M. Nieto-Vesperinas, X. Ding, M. Rahman, T. Zhang, C. Lim, and C.-W. Qiu, "Optical manipulation from the microscale to the nanoscale: fundamentals, advances and prospects," Light Sci. Appl. 6(9), e17039-e17039 (2017).

5. D. Kotsifaki and S. Nic Chormaic, "Plasmonic optical tweezers based on nanostructures: fundamentals, advances and prospects," Nanophotonics 8(2019).

6. D. G. Grier, "A revolution in optical manipulation," Nature 424(6950), 810-816 (2003).

7. J. R. Moffitt, Y. R. Chemla, S. B. Smith, and C. Bustamante, "Recent Advances in Optical Tweezers," Annu. Rev. Biochem. 77(1), 205-228 (2008).

8. M. L. Juan, M. Righini, and R. Quidant, "Plasmon nano-optical tweezers," Nat. Photonics. 5(6), 349-356 (2011).

9. W. D. Phillips, "Nobel Lecture: Laser cooling and trapping of neutral atoms," Rev. Mod. Phys. 70(3), 721-741 (1998).

10. J. Ng, C. T. Chan, P. Sheng, and Z. Lin, "Strong optical force induced by morphology-dependent resonances," Opt. Lett. 30(15), 1956-1958 (2005).

11. J. $\mathrm{Ng}$ and C. T. Chan, "Size-selective optical forces for microspheres using evanescent wave excitation of whispering gallery modes," Appl. Phys. Lett. 92(25), 251109 (2008).

12. J. Ng, R. Tang, and C. T. Chan, "Electrodynamics study of plasmonic bonding and antibonding forces in a bisphere," Phys. Rev. B 77(19), 195407 (2008).

13. J. J. Xiao, J. Ng, Z. F. Lin, and C. T. Chan, "Whispering gallery mode enhanced optical force with resonant tunneling excitation in the Kretschmann geometry," Appl. Phys. Lett. 94(1), 011102 (2009).

14. L.-G. Wang and H.-S. Chai, "Revisit on dynamic radiation forces induced by pulsed Gaussian beams," Opt. Express 19(15), 14389-14402 (2011).

15. B. J. Roxworthy and K. C. Toussaint Jr, "Femtosecond-Pulsed Plasmonic Nanotweezers," Sci. Rep. 2660 (2012).

16. T.-H. Liu, W.-Y. Chiang, A. Usman, and H. Masuhara, "Optical Trapping Dynamics of a Single Polystyrene Sphere: Continuous Wave versus Femtosecond Lasers," J. Phys. Chem. C 120(4), 2392-2399 (2016).

17. L. Pan, A. Ishikawa, and N. Tamai, "Detection of optical trapping of CdTe quantum dots by two-photon-induced luminescence," Phys. Rev. B 75(16), 161305 (2007).

18. J.-l. Deng, Q. Wei, Y.-z. Wang, and Y.-q. Li, "Numerical modeling of optical levitation and trapping of the "stuck" particles with a pulsed optical tweezers," Opt. Express 13(10), 3673-3680 (2005).

19. A. K. De, D. Roy, A. Dutta, and D. Goswami, "Stable optical trapping of latex nanoparticles with ultrashort pulsed illumination," Appl. Opt. 48(31), G33-G37 (2009).

20. N. d. Preez-Wilkinson, A. B. Stilgoe, T. Alzaidi, H. Rubinsztein-Dunlop, and T. A. Nieminen, "Forces due to pulsed beams in optical tweezers: linear effects," Opt. Express 23(6), 7190-7208 (2015).

21. A. Usman, W.-Y. Chiang, and H. Masuhara, "Optical trapping and polarization-controlled scattering of dielectric spherical nanoparticles by femtosecond laser pulses," J. Photochem. Photobiol. A 23483-90 (2012).

22. A. A. Ambardekar and Y.-q. Li, "Optical levitation and manipulation of stuck particles with pulsed optical tweezers," Opt. Lett. 30(14), 1797-1799 (2005).

23. Y. Jiang, T. Narushima, and H. Okamoto, "Nonlinear optical effects in trapping nanoparticles with femtosecond pulses," Nat. Phys. 6(12), 1005-1009 (2010).

24. L. Gong, B. Gu, G. Rui, Y. Cui, Z. Zhu, and Q. Zhan, "Optical forces of focused femtosecond laser pulses on nonlinear optical Rayleigh particles," Photon. Res. 6(2), 138-143 (2018).

25. Y. Zhang, J. Shen, C. Min, Y. Jin, Y. Jiang, J. Liu, S. Zhu, Y. Sheng, A. V. Zayats, and X. Yuan, "NonlinearityInduced Multiplexed Optical Trapping and Manipulation with Femtosecond Vector Beams," Nano Lett. 18(9), 55385543 (2018).

26. M. Hoshina, N. Yokoshi, H. Okamoto, and H. Ishihara, "Super-Resolution Trapping: A Nanoparticle Manipulation Using Nonlinear Optical Response," ACS Photonics 5(2), 318-323 (2018).

27. L. Gong, X. Zhang, B. Gu, Z. Zhu, G. Rui, J. He, Q. Zhan, and Y. Cui, "Optical pulling forces on Rayleigh particles using ambient optical nonlinearity," Nanophotonics 8(6), 1117-1124 (2019). 
28. M. Spector, A. S. Ang, O. V. Minin, I. V. Minin, and A. Karabchevsky, "Temperature mediated 'photonic hook' nanoparticle manipulator with pulsed illumination," Nanoscale Adv. 2(6), 2595-2601 (2020).

29. B. Agate, C. T. A. Brown, W. Sibbett, and K. Dholakia, "Femtosecond optical tweezers for in-situ control of twophoton fluorescence," Opt. Express 12(13), 3011-3017 (2004).

30. J. C. Shane, M. Mazilu, W. M. Lee, and K. Dholakia, "Effect of pulse temporal shape on optical trapping and impulse transfer using ultrashort pulsed lasers," Opt. Express 18(7), 7554-7568 (2010).

31. L.-G. Wang and C.-L. Zhao, "Dynamic radiation force of a pulsed Gaussian beam acting on a Rayleigh dielectric sphere," Opt. Express 15(17), 10615-10621 (2007).

32. J. D. Jackson, Classical electrodynamics (John Wiley \& Sons, 2007).

33. T. Yamaguchi, Y. Okumura, and Y. Amemiya, "Wave impedance in the near field around the fundamental electromagnetic radiating elements," Electron. Comm. Jpn. Pt. I 74(4), 86-95 (1991).

34. J. Butet, P.-F. Brevet, and O. J. F. Martin, "Optical Second Harmonic Generation in Plasmonic Nanostructures: From Fundamental Principles to Advanced Applications," ACS Nano 9(11), 10545-10562 (2015).

35. J. Butet, G. D. Bernasconi, M. Petit, A. Bouhelier, C. Yan, O. J. F. Martin, B. Cluzel, and O. Demichel, "Revealing a Mode Interplay That Controls Second-Harmonic Radiation in Gold Nanoantennas," ACS Photonics 4(11), 2923-2929 (2017).

36. G. Bachelier, J. Butet, I. Russier-Antoine, C. Jonin, E. Benichou, and P. F. Brevet, "Origin of optical secondharmonic generation in spherical gold nanoparticles: Local surface and nonlocal bulk contributions," Phys. Rev. B 82(23), 235403 (2010).

37. J. Butet, S. Dutta-Gupta, and O. J. F. Martin, "Surface second-harmonic generation from coupled spherical plasmonic nanoparticles: Eigenmode analysis and symmetry properties," Phys. Rev. B 89(24), 245449 (2014).

38. G. D. Bernasconi, J. Butet, and O. J. F. Martin, "Dynamics of Second-Harmonic Generation in a Plasmonic Silver Nanorod," ACS Photonics 5(8), 3246-3254 (2018).

39. A. Kiselev, G. D. Bernasconi, and O. J. F. Martin, "Modes interplay and dynamics in the second harmonic generation of plasmonic nanostructures," Opt. Express 27(26), 38708-38720 (2019).

40. J. Zhao, A. O. Pinchuk, J. M. McMahon, S. Li, L. K. Ausman, A. L. Atkinson, and G. C. Schatz, "Methods for Describing the Electromagnetic Properties of Silver and Gold Nanoparticles," Acc. Chem. Res. 41(12), 1710-1720 (2008).

41. B. Gallinet, J. Butet, and O. J. F. Martin, "Numerical methods for nanophotonics: standard problems and future challenges," Laser Photonics Rev. 9(6), 577-603 (2015).

42. A. M. Kern and O. J. F. Martin, "Surface integral formulation for 3D simulations of plasmonic and high permittivity nanostructures," J. Opt. Soc. Am. A 26(4), 732-740 (2009).

43. A. Ji, T. V. Raziman, J. Butet, R. P. Sharma, and O. J. F. Martin, "Optical forces and torques on realistic plasmonic nanostructures: a surface integral approach," Opt. Lett. 39(16), 4699-4702 (2014).

44. T. V. Raziman, W. R. C. Somerville, O. J. F. Martin, and E. C. Le Ru, "Accuracy of surface integral equation matrix elements in plasmonic calculations," J. Opt. Soc. Am. B 32(3), 485-492 (2015).

45. A. M. Kern and O. J. F. Martin, "Pitfalls in the Determination of Optical Cross Sections From Surface Integral Equation Simulations," IEEE Trans. Antennas Propag 58(6), 2158-2161 (2010). 